\title{
Research on Clustering Based Routing Energy-Saving Technology for Wireless Sensor Network
}

\author{
Duo Peng ${ }^{1,2,}$, Suoping $\mathrm{Li}^{1}$ and Ying $\operatorname{Lin}^{1,2}$ \\ ${ }^{1}$ School of Electrical and Information Engineering, Lanzhou University of Technology, Lanzhou 730050, China; ${ }^{2}$ School \\ of Computer and Communication, Lanzhou University of Technology, Lanzhou 730050, China
}

\begin{abstract}
The clustering routing protocol for wireless sensor network (WSN) is the main part of this study. The number of sensor nodes in WSN is numerous and single node is extraordinarily limited in resource. The most significant aim of designing routing protocol of WSN is to reduce the overall energy dissipated in the network and to maximize the network lifetime. After taking into account the transmission and circuit energy together, the appropriate application scenarios of both multiple input multiple output (MIMO) and single input single output (SISO) are derived, and then a collaborative MIMO strategy based on clustering is proposed. Taking the merits of both transmission modes, the strategy reaches an optimal result in respect of energy consumption. Meanwhile, the algorithm distributes the energy consumption of cluster head to collaborative nodes, thus even balance the load and prolong the network lifetime.
\end{abstract}

Keywords: Wireless sensor network, Energy saving, Clustering protocol, Adaptive abdication, Virtual MIMO.

\section{INTRODUCTION}

Wireless sensor network (WSN) is a sensor, data node processing unit and the communication module with a plurality, according to the demand of data collection tasks, a self-organization network. Its mission is to collect data from the environment in which the user is interested. Source node is responsible for collecting data, and the collected data is passed to the sink node (Sink) via a plurality of intermediate nodes in a multi-hop forwarding manner. When normal data passes through intermediate nodes, it requires some processing, removing redundancy by extracting some useful information. According to the application mode, sensor networks can be divided into two types of active (Proactive) and response (Reactive). Active sensor network continuously monitors ambient phenomenon, and sends data at a constant rate; and responsive sensor network transmits data only when the event is triggered $[1,2]$.

Although WSN $\mathrm{s}$ and traditional wireless ad hoc networks have some similarities, yet there are essential differences. That which is mainly reflected in the WSN is a datacentric, a particular type of data acquisition application and design. The traditional wireless ad hoc network is a common platform for a variety of applications and design. WSNs with traditional sensor arrays are essentially different. In the conventional sensor array, data is collected from the individual sensors to a processing center and receives a unified, centralized processing to extract the useful information. In WSN s, the data is refined not only during transmission, but also through a distributed network processing, and is constantly being refined to extract useful information.

*Address correspondence to this author at the School of Electrical and Information Engineering, Lanzhou University of Technology, Lanzhou 730050, China; E-mail: pengduo7642@163.com
Wireless transmission of energy mainly comes from the wireless data transceiver modules and power consumption when idle listening. In the process of running the network, the wireless module can be in four states: send, receive, idle and sleep. And in these four states, energy amount used is not the same. Fig. (1) shows a comparison of several WSNs' energy consumption amount. The main reference to the energy consumption comparison chart [3-5] is obtained mainly for short-distance WSN transmission $(10-100 \mathrm{~m})$ scenario, which sending and receiving energy consumption is comparable. Under this scenario sending and receiving energy consumption is comparable.

Fig. (1) shows sensing energy mainly for low-power sensor; when considering the application of video capture sensor, the sensor power consumption cannot be ignored. But since the object of this paper is a traditional sensor network, therefore, sensing energy consumption has been ignored.

As can be seen from Fig. (1), the wireless module is the primary energy source. The power consumption of wireless module in idle state is almost equal to the energy received state. In order to effectively save the energy, it should be possible to make a wireless module sleep. Wireless module energy mainly comes from the transmission circuit, amplifier and receiver circuit.

\section{ANALYSIS AND STUDY OF ALGORITHM FOR LEACH}

\subsection{Clustering Hierarchical Routing Algorithm}

WSNs are with flexible communication infrastructure or ad hoc features, so that it has a great deal of flexibility. Controlled distribution, no center, and features such as dynamic topology limit the scalability of the system. The design using clustering algorithm for WSN is divided into logical hierarchies. This hierarchy can help reduce maintenance overhead, 


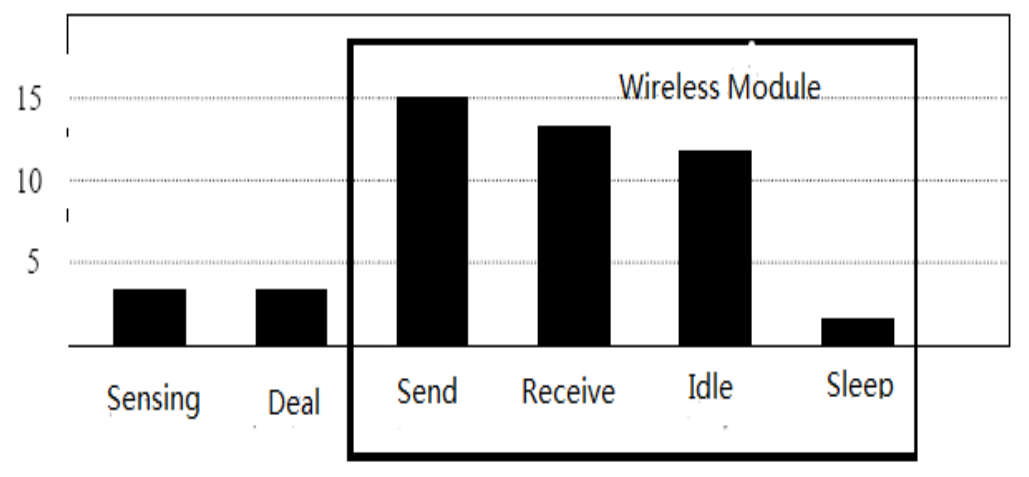

Fig. (1). Contrast of energy consumption.

improve network scalability and improve channel spatial reuse. On a large-scale, network-oriented application system is important.

Clustering algorithms of tasks in a collection node have been divided into several smaller collections that do not intersect or meet each other. They are clustered within and between cluster nodes to form a two-layered structure. These fine-grained clusters are drawn to form a nested hierarchy. More hierarchies are applied to larger networks, but generally a two-story structure has to meet most application needs. LEACH and improved algorithm are generally used in twotier clustering models.

Using the above information, this level-based hierarchical routing algorithm structure has the following advantages: The number of nodes' reduced participation in the routing computation, a reduced routing table size, reduces communication overhead to exchange routing information required to maintain routing tables and the memory overhead required. A more stable sub network can be selected through the algorithms, thus reducing the effect of topology changes brought to the routing algorithm. Topology algorithm can be easily passed all kinds of information to the sink node through the management and the cluster nodes, such as energy, security, fault etc. In addition, the sink node can effectively give command to the other nodes in the network sent through the cluster head node, which is the flat routing that can't be effectively realized.

The hierarchical model of mathematics is described as follows: Assuming the computing and communication capabilities of WSN node plane is exactly the same, there is no existence of one-way link. Then the topology of WSNs can be abstracted as a connected graph $\mathrm{G}=(\mathrm{V}, \mathrm{E})$, in which $\mathrm{V}$ denotes the set of nodes in the network, $M$ is the number of nodes, $\mathrm{E}$ is the two-way link between two nodes set (the set of direct communication ability among all of the nodes clusters). For graph $\mathrm{G}=(\mathrm{V}, \mathrm{E})$, a positive integer $\mathrm{M}, 1 \leq \mathrm{V} \leq \mathrm{M}$, then a $\mathrm{L}(\mathrm{L} \geq 1)$ layers network structure model is the set of the $\mathrm{V}$, which can be expressed as $V=\left\{P_{1}, \mathrm{P}_{2}, \ldots \ldots . \mathrm{P}_{l}\right\}$, representing the nodes collection of the layer $i$; it is obvious that

$V=\sum_{\mathrm{i}=1}^{1} \mathrm{P}_{i} \cdot$

The nodes collection of the layer $\mathrm{i}$ is $P_{i}=\left\{v_{1}, v_{2}, \ldots \ldots v_{x}\right\}$, $C_{i}=\left\{v_{i j}\right\}$ represents a node set of cluster $\mathrm{i}$, where $v_{i 0}$ repre- sents the cluster head node, and $v_{i j}$ represents normal node. Then

$$
P_{i}=\sum_{i} C_{i},
$$

for layer $P_{i}$ any one of the cluster structures can meet the following conditions:

A) $\bigcup_{i=1}^{n} C_{i}=P_{i}$, each of the nodes belongs to different clusters;

B) $C_{i} \cap C_{j}=\phi$, no overlap between any two cluster;

C) $C_{i} \geq 2$, any number of nodes in a cluster is greater than or equal to 2 .

Typical applications of WSN mode include sending information to the base station after each sensor node to collect data. However, in many cases, this model for energyconstrained sensor nodes is unacceptable. Further, because between many of the neighboring sensor data collected there is great compatibility, some means of data fusion can be used and then the data is transmitted to the base station. Under the guidance of this goal, it will automatically generate network clustering algorithm thinking. Clustering idea is to select a few nodes in the network as a cluster head, whereas the rest of the sensor nodes will serve as a cluster head member. Each member of the collected data is not sent directly to the base station, but to the cluster-head. After the first cluster member is sent to the cluster-head to collect data for compression, it is sent to the base station. Utilization of clustering algorithm by reducing the number of times each node sends data to the base station, leads to greatly reducing the energy consumption of each node. And the number of nodes participating in the network channel contention, leads to improving the channel. LEACH algorithm is a classical clustering algorithm.

\subsection{Mathematical Description of the WSN Routing}

As mentioned above, WSNs can be represented by figure $\mathrm{G}=(\mathrm{V}, \mathrm{E})$. A cost function $\mathrm{C}$ is defined as $\mathrm{E}: \mathrm{C}=\mathrm{f}(\mathrm{N}, \mathrm{B}$, $\mathrm{T}$ ), of which $\mathrm{N}$ indicates the data transmission unit energy consumption cost, B represents the path of the remaining channel capacity (bandwidth), and T represents the average delay time (ms) path. WSN routing problem can be abstracted by the energy constrained by optimization. So WSN rout- 
ing is essentially looking for the minimum cost of the transmission path in the limited energy supply scenario $[6,7]$ :

$\mathrm{G}=\min \sum_{j=1}^{\mathrm{N}} C(\mathrm{k})+\mathrm{P}(\mathrm{k})$

Of which, $\mathrm{k}$ represents the number of hops in the path, $N_{\max }$ represents the maximum energy of nodes, $\mathrm{P}(\mathrm{k})$ represents the cost of each path for routing node processing the packet, $P(\mathrm{k})=\mathrm{kb}+\mathrm{k} / \mathrm{u}, \mathrm{b}$ represents the unit cost of forwarding packets, $\mathrm{u}$ represents the cost of processing unit packets.

\section{VIRTUAL COLLABORATIVE MIMO ALGO- RITHM BASED CLUSTERING}

\subsection{Design Ideas and Difficulty}

As can be seen from the foregoing description, MIMO system has advantages such as the channel capacity and antifading properties, making it a powerful alternative to the sensor network design. The only problem is that, it is difficult to construct large multiple antennas technically in very small sensor nodes. And even if such a node is realized, it cannot meet the requirements of the spatial diversity of the distance between antennas as the distance between multiple antennas is too close. Some policy-based virtual MIMO single antenna nodes have solved this problem. Further, considering the energy consumption by system nodes, multiantenna MIMO system structure will inevitably lead to more energy consumption by the system [8-10]. This is one of the factors that need to be considered in the design of MIMO policies.

The main contribution of this paper is to propose a clustering algorithm based on network thinking. The algorithm takes optimization energy as target. Taking into account the total energy consumption, including node system, and taking the utilization of MIMO and SISO to their advantage, we ensure the optimum design of a clustering algorithm to achieve the energy optimization on the premise of achieving quality communication. In MIMO systems, the encoding used is Alamouti STBC as mentioned in the previous section $[11,12]$.

\subsection{Energy Model of MIMO System}

Compared with the conventional single antenna SISO system, using the same transmission power and bit error rate under the standard load, multiple input multiple output (MIMO) system can support a higher transmission rate. That is, at the same throughput and bit error rate, MIMO system's consumed energy is smaller than the SISO.

For the sensor network, first analyzed its transmit power $P_{t}$ as follows:

$P_{t}=\left(1+\mathrm{P}_{\text {out }}\right)$

Of which, $P_{\text {out }}$ is energy transmission rate, $\alpha=\xi / \eta-1, \xi$ depends on the modulation scheme and constellation size, $\eta$ reflects the ratio of the signal peak and average. $\mathrm{H}$ is constant related with RF power amplifier hardware.

According to the link load relationship:
$\mathrm{P}_{\text {out }}=\frac{(4 \pi)^{2} d^{2} M_{t} N_{f}}{G_{t} G_{r} \lambda^{2}} \bar{E}_{b} R_{b}$

Of which, $\mathrm{d}$ is transmission distance. $G_{t}$ and $G_{r}$ are the antenna gain of the sender and receiver respectively. $E_{b}$ is the energy-per-bit required for the receiving side that guaranteed reaches a certain bit error rate. $R_{b}$ is bit transfer rate. $M_{t}$ is used to compensate for background noise and interference link idle. $N_{f}$ is the receiver noise factor.

From the foregoing formula:

$\mathrm{P}_{t}=(1+\partial) \frac{(4 \pi)^{2} d^{2} M_{t} N_{f}}{G_{t} G_{r} \lambda^{2}} \overline{E_{b}} R_{b}$

Assuming the channel attenuation matrix is $\mathrm{H}$, and transmitting antenna powers $M_{t}$ are equal. By the known formula, the receiver SNR is

$\tau_{b}=\frac{\overline{E_{b}} / M_{t}}{N_{0}}\|H\|_{F}^{2}$.

Using BPSK modulation mode, the average error rate is found as follows: $\overline{P_{b}}=Q\left(\sqrt{2 \tau_{b}}\right)$. According to Ghernoff boundary theorem :

$\overline{P_{b}}=\left(\frac{\overline{E_{b}}}{M_{t} N_{0}}\right)^{-M_{\mathrm{t}}}$

The $\overline{E_{b}}$ boundary value is:

$\overline{E_{b}} \leq \frac{M_{t} N_{0}}{\bar{P}_{b}^{1 / M_{\mathrm{t}}}}$

Taking the boundary values equal, from equations (4) and (6) we get:

$\mathrm{P}_{t}=(1+\partial) \frac{M_{t} N_{0}}{P_{b}^{1 / M_{\mathrm{t}}}} \frac{(4 \pi)^{2} d^{2} M_{t} N_{f}}{G_{t} G_{r} \lambda^{2}} R_{b}$

Therefore, the transmission power per bit is :

$\mathrm{E}_{b t}=\frac{P_{t}}{R_{b}}=(1+\partial) \frac{M_{t} N_{0}}{P_{b}^{1 / M_{\mathrm{t}}}} \frac{(4 \pi)^{2} d^{2} M_{t} N_{f}}{G_{t} G_{r} \lambda^{2}}$

Numerical method can obtain a more accurate value of $\overline{E_{b}}$. From equation (8) it can be seen that $\mathrm{E}_{b t}$ is reduced with the increase in the number of antennas $M_{t}$ and low, that is, MIMO diversity gain advantage is reflected. From equation (7), assume:

$$
P=\alpha\left(M_{t}\right) \cdot d^{2}
$$

Using equation (7), it can be verified with Matlab that $\alpha\left(M_{t}\right) \underline{\text { is }}$ the decreasing function of $M_{t}$ in interval $[1, \mathrm{n}]$, where $P_{b}$ is the smaller, $\mathrm{n}$ is the greater. For example, when $P_{b}=0.05, \mathrm{n}=3$; and when $P_{b}=0.001, \mathrm{n}=8$. Thus, when $\mathrm{J}$ is an integer greater than 1 and less than $\mathrm{n}$, there must be $\alpha(\mathrm{J})<\alpha(1)$.

In order to compare the energy consumption of MIMO and SISO, given the introduction of the system parameters, 2 $\times 2$ MIMO system and SISO system transmit power of per bit comparison result is shown in Fig. (2) (from equation (8)). 


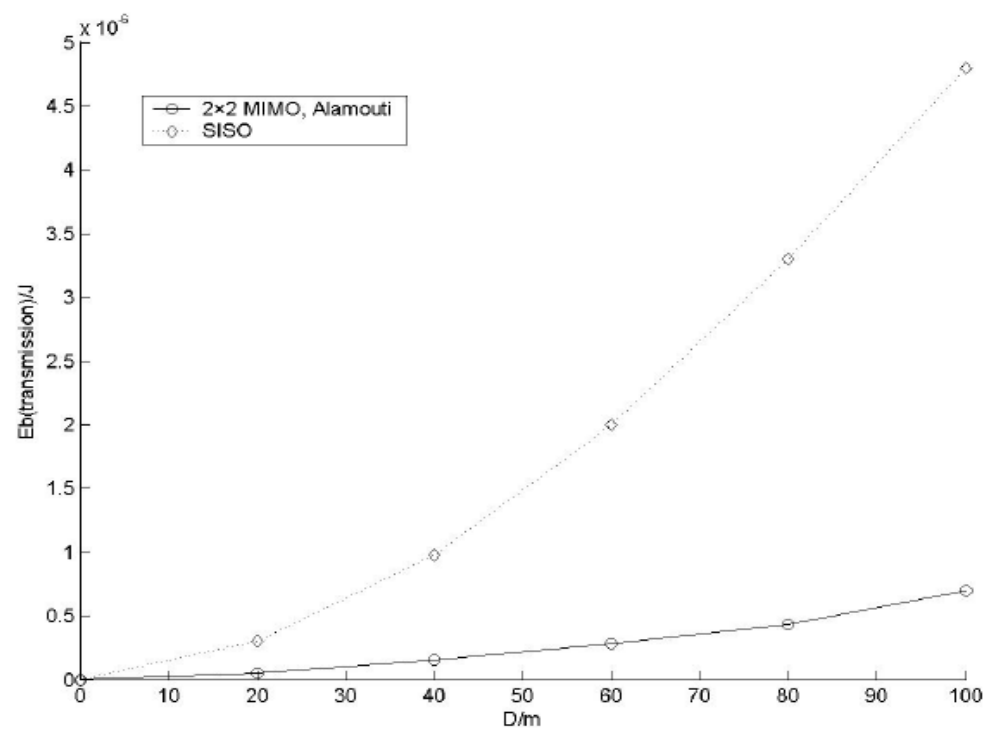

Fig. (2). Transmission energy per Bit, MIMO versus SISO.

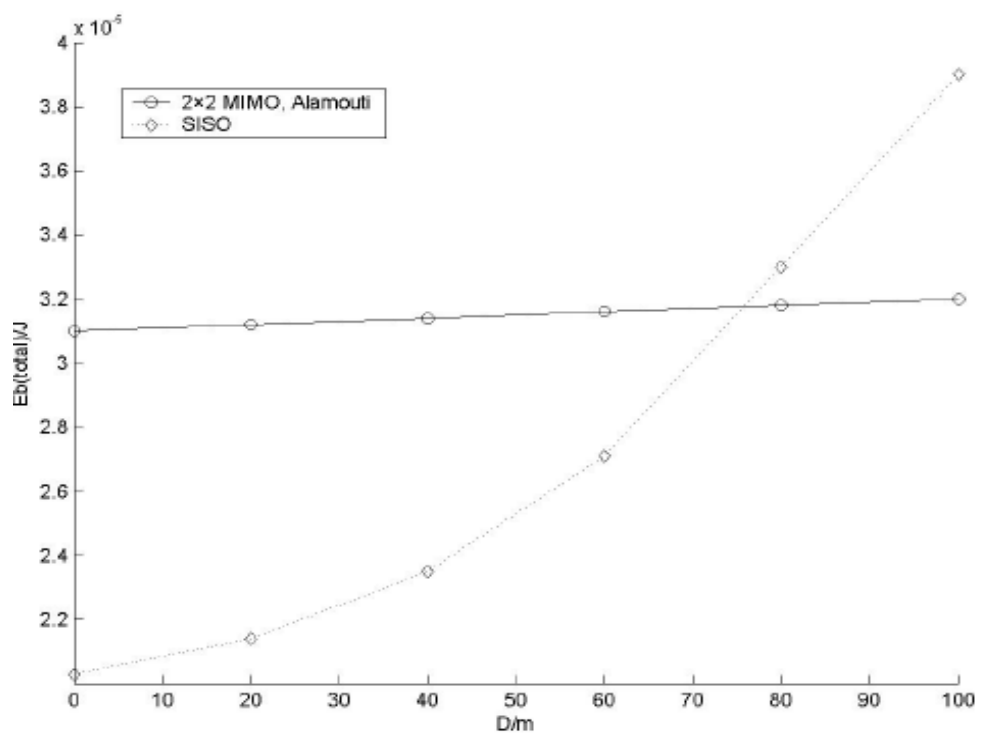

Fig. (3). Total energy consumption over d, MIMO versus SISO.

As can be seen from Fig. (2), MIMO always consumes less energy compared to single input single output (SISO) $[13,14]$.

However, the application of such a sensor is mainly attributed for short distance transmission, that is the system energy consumption is also a non-negligible factor. The system energy consumption $P_{c}$ can be approximated as:

$$
\begin{aligned}
& P_{c}=M_{t}\left(\mathrm{P}_{D A C}+\mathrm{Pmix}+\mathrm{P}_{f i l t}\right)+2 P_{s y n} \\
& +M_{r}\left(\mathrm{P}_{L N A}+\mathrm{P}_{m i x}+\mathrm{P}_{I F A}+\mathrm{P}_{f i l}+\mathrm{P}_{A D C}\right)
\end{aligned}
$$

Of which $\mathrm{P}_{D A C}$, Pmix $, \mathrm{P}_{\text {filt }}, \mathrm{P}_{L N A}, \mathrm{P}_{\text {mix }}, \mathrm{P}_{I F A}, \mathrm{P}_{\text {filr }}, \mathrm{P}_{A D C}$ and $P_{s y n}$ represent the digital to analog converter, a mixer, a low noise amplifier, an intermediate frequency amplifier, transmitting and receiving side filter, analog to digital converter and the power consumed by the synchronizer respectively.
Since more nodes are involved in the MIMO system communication process, therefore the system must consume more energy. After considering the energy consumption rate of the system, a comparison of MIMO system and SISO system energy consumption is given in Fig. (3) (from equation (8) and (10)). As can be seen from Figs. (4, 5), in sensor networks, MIMO system has a greater advantage in longdistance communication $(>75 \mathrm{~m})$. This is due to multipath fading, the nature of its antenna diversity gain. And in the short-distance transmission, the system's energy consumption has become a major factor. In lieu of these characteristics, a rather more energy-efficient network can be designed based on MIMO system, thus extending the network lifetime.

\subsection{Virtual MIMO Algorithm-Based Clustering}

As already discussed earlier, the clustering algorithm's strengths include: the location of the base station is generally 


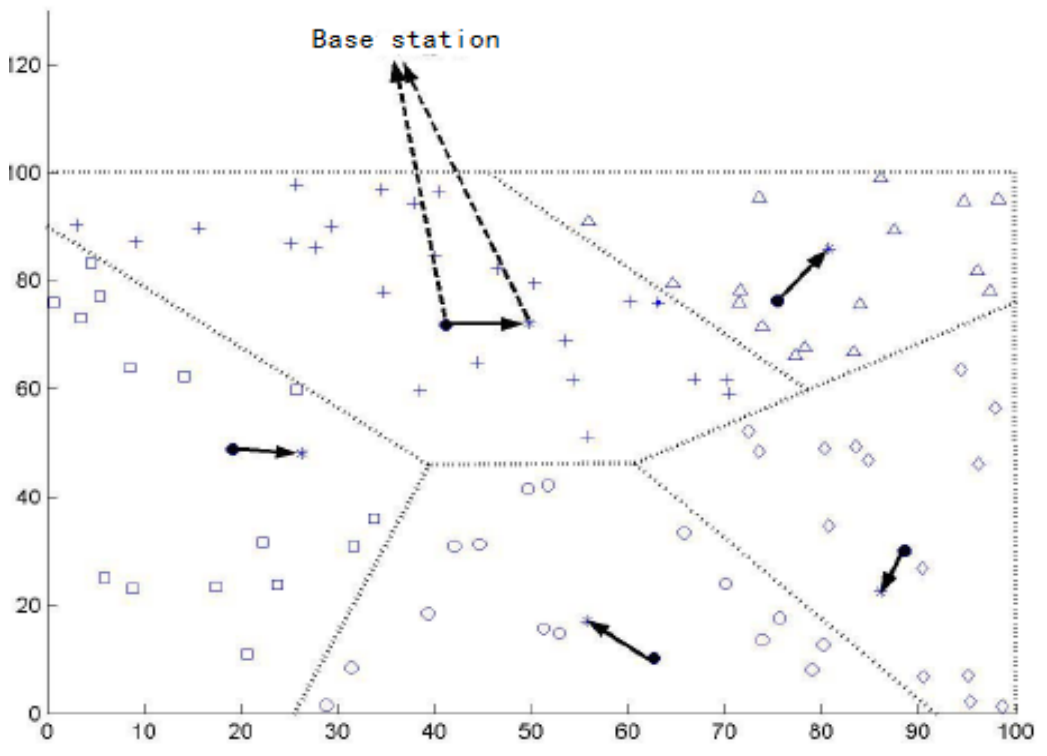

Fig. (4). Illustration of Virtual MIMO Algorithm.

fixed; and the distance between control points is usually far. However, because there are more local transmission sensors distributed in close proximity to each other, so the channel is assumed as AWGN channel. As can be seen, the virtual MIMO technology is ideally suited for such a clustering structure. As the WSN is difficult to control centrally, therefore the difficulty lies in how to construct efficient transmission distributed algorithm ideas.

Algorithm is as follows:

A. Cluster formation stage: First, each node passes through a distributed algorithm decides itself whether it serves as the cluster head during the current round, by becoming a cluster head node to broadcast information to surrounding nodes. However, the cluster head node cannot send and receive the broadcast information from more than one cluster heads, it will choose the most suitable cluster head to join the cluster (i.e. accordance to the principle of the nearest one). Then it will feedback information including the required value of the residual energy of nodes and cluster nodes itself.

B. MIMO Antenna selection phase: After the formation of clusters, cluster head brought energy information to get in all the $\mathrm{N}$ cluster members. Assuming energy of the $\mathrm{k}$-th member is $E_{k}(1 \leq \mathrm{k} \leq \mathrm{N})$, the algorithm also can help determine the distance $d_{k}$ of the member by the strength of the received signal. Cluster heads choose the appropriate number of member nodes to form a virtual MIMO antenna array, these nodes are called cooperative nodes.

C. Slot table allocation phase: Cluster head creates a TDMA slot table, to assign a data transmission time slot for all members of the cluster (including cooperative nodes). In addition, at the end of the table cooperative nodes are joined with ID number, the last node joined with its own cluster head ID as the cluster flag, then the table is broadcast. Members can be determined after receipt of the tables whether they fit cluster slot table, and whether they are selected as the cooperative nodes.
D. The data acquisition phase within the cluster: All ordinary nodes and nodes in collaborative arrangements slot table, send collected data to the cluster head in their own time slot. The rest of the time they enter dormancy. The first cluster continues to work. Cooperative nodes automatically wake up in the slot table specified stage, waiting for the integrated data's reception by the cluster head.

E. Remote communication phase: After the round, cluster heads collect information from all clusters, perform data fusion, and then the information is sent to the K-1 cooperative nodes. Each node coordinates synchronization and encoding. Finally, this collaboration will send data to a remote cluster of multi-antenna base station system, which is part of the MIMO transceiver system.

\section{ALGORITHM SIMULATION}

\subsection{NS2 Simulation Environment}

NS2 software and LEACH algorithm expansion packs are used for building simulation environment.

\subsection{Simulation Scenarios and Parameters}

The simulation environment includes a network of 100 nodes evenly distributed in the random $(x=0, y=0)$ to square $(x=50, y=50)$ area. The base station is located in the random location. Head cluster average number is 5. Each node transmits a packet length of 2000bits. After data fusion, cluster head packet length becomes 10kbits. Simulation is performed using Monte-Karol statistical experimental averaging method.

\subsection{The Simulation Results and Analysis}

Fig. (5) shows a comparison of network lifetime. The figure shows that the virtual MIMO proposed algorithm has a longer network lifetime than LEACH algorithm. When $\mathrm{J}=$ 2 , the network lifetime is extended by $30 \%$. The greater the $\mathrm{J}$ values, the longer the network lifetime is. However, the 


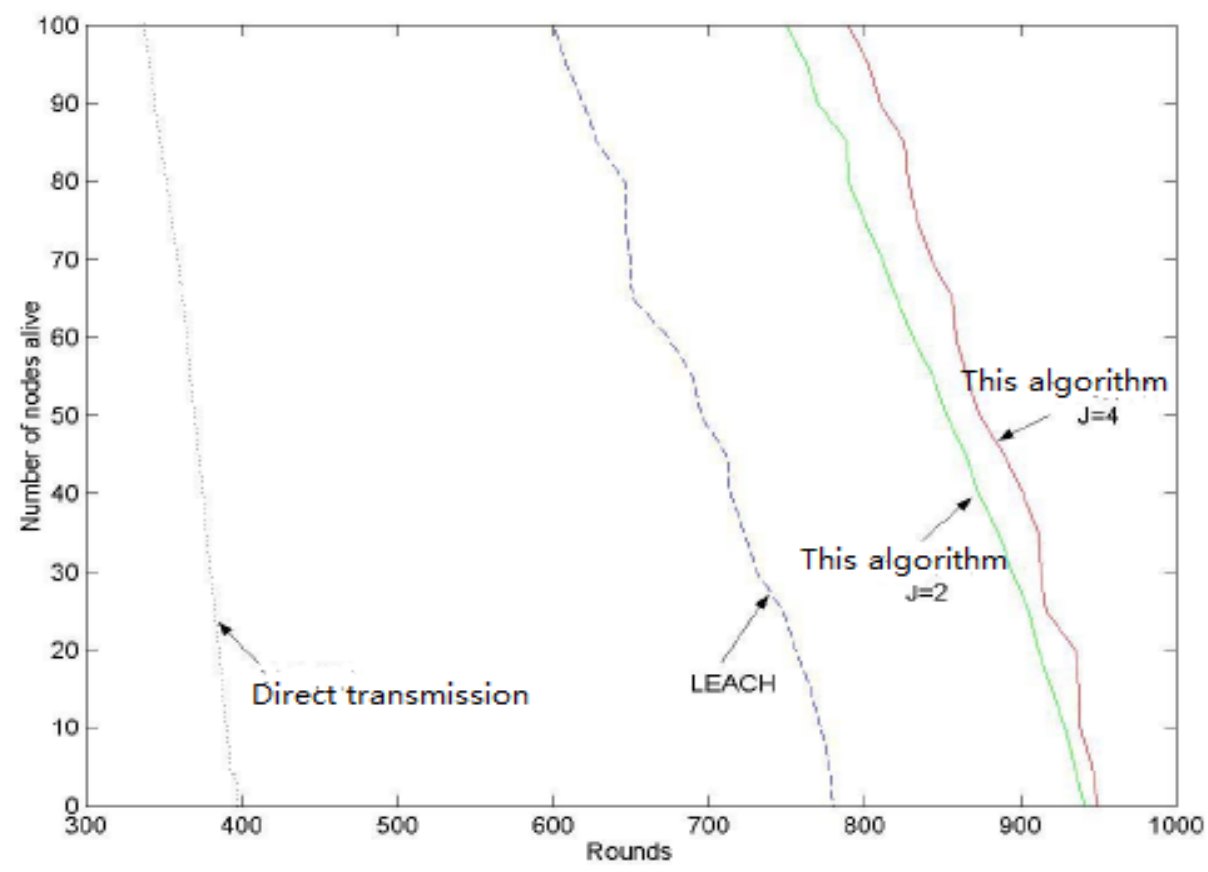

Fig. (5). Comparison of network lifetime.

higher the complexity of the encoding and decoding, the greater the complexity of the algorithm is.

\section{CONCLUSION}

This paper establishes a clustering algorithm model for efficient energy consumption based on MIMO algorithms communication, which is compared with the traditional single-antenna LEACH energy model. A comparative analysis is provided on the basis of the application of collaborative MIMO technology. Simulation results show that the algorithm, without compromising on the quality of communication, is more energy efficient than the original SISO transmission. At the same time, through the virtual MIMO strategy, a lot of energy is released that is consumed by the original cluster head node in shared collaboration, which further balances the network load and extends the network lifetime.

\section{CONFLICT OF INTEREST}

The authors confirm that this article content has no conflict of interest.

\section{ACKNOWLEDGEMENTS}

Funding for this study by Gansu Province Natural Science Foundation (No: 1308RJZA104) and National Natural Science Foundation of China funding (No: 61363078) is greatly acknowledged.

\section{REFERENCES}

[1] I. F. Akyildiz, S. Weilian, Y. Sankarasubramaniam, E. Cayirci. "A survey on sensor networks," IEEE Communications Magazine, 2002, 40(8), 102 114.

[2] J. A. Stankovic, T. E. Abdelzaher, L. Chenyang, S. Liu, J. C. Hou. "Real-time communication and coordination in embedded sensor networks," In: Proceedings of the IEEE, vol. 91, no. 7, pp. 1002 1022, 2003.
[3] J. M. Kahn, R. H. Katz, and K. S. Pister, "Next century challenges: mobile networking for "Smart Dust," In; Proceedings of the 5th Annual Joint ACM/IEEE International Conference on Mobile Computing and Networking (MOBICOM'99), Seattle, A, USA, New York, NY: ACM Press. pp. 271 278, 1999.

[4] C. Chee-Yee, and S. P. Kumar, "Sensor networks: evolution, opportunities, and challenges," In: Proceedings of the IEEE, 2003, 91(8): 1247 1256

[5] V. Raghunathan, C. Schurgers, S. Park, M. B. Srivastava. "Energyaware wireless micro-sensor networks," IEEE Signal Processing Magazine, vol. 19, no. 2, pp. 40 50, 2002.

[6] T. Pering, T. Burd, and R. Brodersen, "The simulation and evaluation of dynamic voltage scaling algorithms," In: Proceedings of the ACM International Symposium on Low Power Electronics and Design, Monterey, CA, USA, New York, NY: ACM Press, pp. 76 81, 1998.

[7] A. Sinha, A. Wang, and A. P. Chandrakasan, "Algorithmic transforms for efficient energy scalable computation" Proceedings of the ACM International Symposium on Low Power Electronic Design (ISLPED2000), Rapallo, Italy, New York, NY: ACM Press, pp.31 36, 2000.

[8] C. F. Chiasserini, "On the concept of distributed digital signal processing in wireless sensor networks," In: Proceedings of the IEEE Military Communications Conference (MILCOM'02), Anaheim, CA, USA, Piscataway, NJ: IEEE Press, pp. 260 264, 2002.

[9] J. Ed Callway, "A communication protocol for wireless sensor networks: [Doctoral Dissertation]," Boca Raton, Florida: University of Florida Atlantic, 2002.

[10] W. Ye, J. Heidemann, and D. Estrin, "Medium access control with coordinated adaptive sleeping for wireless sensor networks." IEEE/ACM Transactions on Networking, vol. 12, no. 3, pp. 493 506, 2004,.

[11] G. Lu, B. Krishnamachari, and C. S. Raghavendra, "An adaptive energy-efficient and low-latency MAC for data gathering in wireless sensor networks," In: Proceedings of the 18th IEEE International Parallel and Distributed Processing Symposium (IPDPS'04), Santa Fe, NM, USA, Los Alamitos, CA: IEEE Computer Society, pp. 224 232, 2004.

[12] B. H. Liu, C. T. Chou, J. Lipman, S. Jha. "Using frequency division to reduce MAI in DS-CDMA wireless sensor networks," In: Proceedings of the IEEE Wireless Communications and Networking Conference (WCNC'05), New Orleans, USA, IEEE Press, pp. 657 663, 2005. 
[13] C. Shuguang, and R. Madan, “Joint Routing, MAC, and Link Layer Optimization in Sensor Networks with Energy Constraints" In: Proceedings of the IEEE International Conference on Communications (ICC), South Korea, IEEE Press, pp. 725 729, 2005.
[14] A. El Gamal, C. Nair, B. Prabhakar, E. Uysal-Biyikoglu, S. Zahedi. "Energy-efficient scheduling of packet transmissions over wireless networks" In: Proceedings of the IEEE 21st Information Communications Conference (INFOCOM'02), New York, NY, USA, Piscataway, NJ: IEEE Press, pp. 1773 1782, 2002.

Received: September 16, 2014

Revised: December 23, 2014

Accepted: December 31, 2014

(C) Peng et al.; Licensee Bentham Open.

This is an open access articles licensed under the terms of the Creative Commons Attribution-Non-Commercial 4.0 International Public License (CC BY-NC 4.0) (https://creativecommons.org/licenses/by-nc/4.0/legalcode), which permits unrestricted, non-commercial use, distribution and reproduction in any medium, provided that the work is properly cited. 